\title{
Astrocytes from Cerebral Cortex or Striatum Attract Adult Host Serotoninergic Axons into Intrastriatal Ventral Mesencephalic Co-Grafts
}

\author{
Audrey Petit, Philippe Pierret, Annie Vallée, and Guy Doucet \\ Département de Pathologie et Biologie Cellulaire and Centre de Recherche en Sciences Neurologiques, Faculté de \\ Médecine, Université de Montréal, Montréal, Québec, Canada H3C 3J7
}

The identification of axon growth inhibitory molecules offers new hopes for repair of the injured CNS. However, the navigational ability of adult CNS axons and the guidance cues they can recognize are still essentially unknown. Astrocytes may express guidance molecules and are known to have different regional phenotypes. To evaluate their influence on the affinity of adult serotoninergic (5-HT) axons for a projection target, we co-implanted astrocytes from the neonatal striatum, cortex, or ventral mesencephalon together with fetal ventral mesencephalic tissue into the striatum of adult rats. Two months after surgery, quantification after in vitro $5-\left[1,2-{ }^{3} \mathrm{H}\right]$ serotonin $\left(\left[{ }^{3} \mathrm{H}\right] 5-\right.$ HT) uptake and autoradiography showed that ventral mesencephalic grafts with co-grafted cortical or striatal astrocytes were four times and three times, respectively, more densely innervated by host 5-HT axons than control ventral mesencephalic grafts with or without co-grafted ventral mesencephalic astrocytes. Immunohistochemistry for glial fibrillary acidic protein, vimentin, or chondroitin-sulfate proteoglycans revealed no qualitative or quantitative differences in host astroglial scar or production of inhibitory molecules that could explain these differences in 5-HT innervation. These results demonstrate that astrocytes grown in culture from different brain regions have the potential to influence the growth and maintenance of adult 5-HT axons in a graft of neural tissue from another brain region. It should now be feasible to identify the molecules expressed by cultured cortical or striatal, but not by ventral mesencephalic, astrocytes that have these tropic actions on 5-HT axons of the neostriatum.

Key words: axon guidance; regeneration; astrocyte; neural transplantation; cell culture; serotonin neurons; neostriatum; substantia nigra
Recent progress in the identification of axon growth inhibitory molecules (McKeon et al., 1991; McKerracher et al., 1994; Mukhopadhyay et al., 1994; Chen et al., 2000; GrandPre et al., 2000) and treatments to counteract their activity (Huang et al., 1999) shows that regeneration in the injured CNS is foreseeable. Nevertheless, the navigational capabilities of axons in mature CNS, information that will be useful to optimize functional recovery, remain essentially unknown.

Neural transplantation experiments demonstrated that fetal neurons may form appropriate connections in mature CNS (Wictorin et al., 1991; Deacon et al., 1994; Isacson and Deacon, 1996), but the identity of guidance cues used by growing axons is unknown. Progress was made in the identification of guidance molecules acting during development (Tessier-Lavigne and Goodman, 1996), but it is unclear whether and where they are expressed in adult CNS and what types of adult neurons may recognize them.

Fetal neural tissue transplantation into the CNS has been used

\footnotetext{
Received Feb. 14, 2001; revised June 21, 2001; accepted July 6, 2001.

This work was supported by the Canadian Institutes of Health Research (Grant MT-15119) and by studentships from the Groupe de Recherche sur le Système Nerveux Central [Fonds pour la Formation de Chercheurs et l'Aide à la Recherche (FCAR), Centres de Recherche] to A.P. and P.P., a studentship from the Fonds de la Recherche en Santé du Québec (FRSQ)/FCAR-Santé to A.P., and a scholarship from the FRSQ to G.D. We thank Dr. Laurent Descarries for critical revision of this manuscript and Denis Rodrigue for technical assistance.

Correspondence should be addressed to Dr. Guy Doucet, Département de pathologie et biologie cellulaire and Centre de recherche en sciences neurologiques, Faculté de médecine, Université de Montréal, C.P. 6128, Succursale Centre-ville, Montréal, Québec, Canada H3C 3J7. E-mail: guy.doucet@umontreal.ca. Copyright (ㄷ) 2001 Society for Neuroscience 0270-6474/01/217182-12\$15.00/0
}

to study the plasticity of adult host neurons (Oblinger and Das, 1982; Castro et al., 1988; Doucet et al., 1989; Sørensen et al., 1990). This approach was used to study the response of adult serotoninergic (5-HT) neurons to guidance cues. These neurons readily innervate homotypic neural grafts in hippocampus, striatum, or thalamus (Azmitia et al., 1981; Wictorin et al., 1988; Nothias et al., 1990; Labandeira-Garcia et al., 1991; Lu et al., 1991) but project barely into intrastriatal ventral mesencephalic (VM) grafts (Doucet et al., 1989; Mounir et al., 1994), although many individual dorsal raphe 5-HT neurons send collaterals to both VM and neostriatum in normal brain (Van der Kooy and Hattori, 1980) and should therefore recognize both targets. Indeed, grafts of striatal tissue receive four to five times more 5-HT innervation than VM grafts (Pierret et al., 1998b). Serotonin axons innervating striatum may prefer striatal tissue, an affinity likely mediated by surface receptors. Because neural grafts include astrocytes and neurons, both cell types may express molecules recognized by such receptors. Astrocytes express axon guidance molecules (Smith et al., 1986; Steindler et al., 1990; Laywell and Steindler, 1991; Powell et al., 1997) and differ among brain regions (Denis-Donini et al., 1984; Denis-Donini and Estenoz, 1988; Liu and Lauder, 1992; Vickland and Silver, 1996).

To assess the influence of astrocytes on the ability of adult 5-HT axons to grow into a VM graft, we co-grafted fetal VM tissue with astrocytes cultured from the cerebral cortex, striatum, or VM into the neostriatum of adult rats (see Fig. 1). With a technique based on autoradiography after uptake of 5-[1,2$\left.{ }^{3} \mathrm{H}\right]$ serotonin $\left(\left[{ }^{3} \mathrm{H}\right] 5-\mathrm{HT}\right)$ in brain slices (Doucet and Descarries, 1993), we quantified the number of 5-HT axonal varicosities in 


\section{Dissection and culture of neonatal astrocytes}

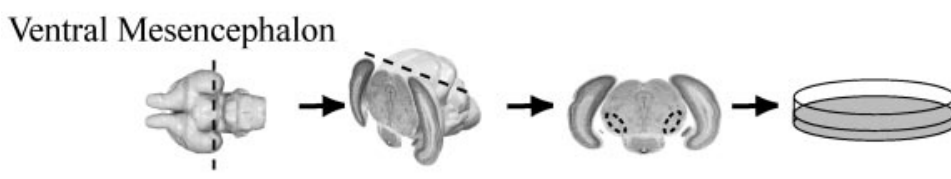

Neostriatum
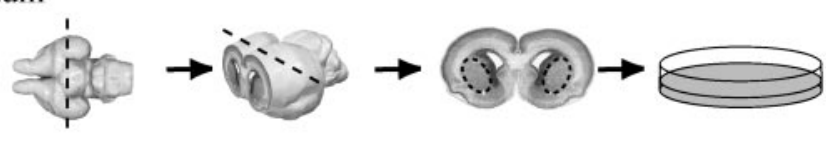

Cerebral Cortex

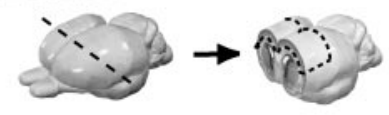

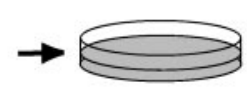

\section{Co-implantation of cultured astrocytes with ventral mesencephalic (or striatal) tissue}

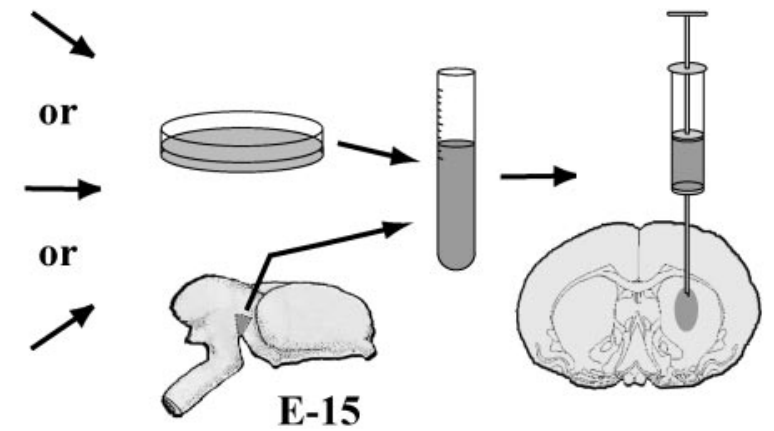

Figure 1. Dissection and culture of neonatal astrocytes, mixing with fetal VM tissue, and co-implantation into adult rat neostriatum. Astrocytes were dissected from the frontoparietal cortex, neostriatum, or substantia nigra of P0 rat pups. At confluence, $22 \mathrm{~d}$ later, they were labeled by incubation with Hoechst fluorescent dye, dissociated with trypsin, mixed with fetal VM (or lateral ganglionic eminence), and implanted into the neostriatum of adult rats. The recipients were killed 10-40 d, 2 months, or 4 months later, as summarized in Table 1. Brain specimen drawings were adapted from the atlas of Altman and Bayer (1995).

the implants. Co-grafted astrocytes were labeled with Hoechst vital dye, before mixing with VM tissue, to monitor their distribution. Immunohistochemistry for several neural markers and axonal tracing were used to characterize cultures, implants, and host projections into the implants. Striatal implants, with or without co-grafted VM astrocytes, were also examined as controls (see Table 1).

\section{MATERIALS AND METHODS}

Experimental animals. All experimental procedures were approved by the Ethics Committee on the use of experimental animals of the Université de Montréal (Protocol 99102). Adult female Sprague Dawley rats $(n=$ $133 ; 200-225 \mathrm{gm}$ at the time of transplantation surgery) were used as recipients for transplantation. Surgery was performed under fentanyl/ fluanisone (Hypnorm; Janssen, Beerse, Belgium)/midazolam (Versed; Hoffman-Laroche, Mississauga, ON) anesthesia (1:1 with equal volumes of distilled water; $0.27 \mathrm{ml} / 100 \mathrm{gm}$ ) (Flecknell, 1996). Forty-six litters of embryonic day (E) 15 (crown-rump length $11 \mathrm{~mm}$; VM tissue), and two litters of E16 (striatal tissue) Sprague Dawley rat fetuses from deeply anesthetized dams (Hypnorm-Versed) were used as donors. In each session, one-third of the fetal neural tissue pieces were used to prepare control single grafts (VM or striatal tissue), whereas the rest were mixed with one or the other type of cultured astrocytes for co-grafting. Implants were prepared in a total of 24 surgical sessions, i.e., 5-8 separate transplantation sessions for each experimental group, to ensure that variations in culture or tissue preparation would affect all groups at random.

Preparation and immunocytochemical characterization of cultured astrocytes. Purified, type 1 astrocytes were obtained from frontoparietal cortex, neostriatum, and VM of newborn rats (day of birth, P0) (Fig. 1) by the method of McCarthy and De Vellis (1980). Brain tissue was dissected, meninges were stripped off, and cells were dissociated by incubation with gentle stirring in HBSS (Life Technologies-BRL, Gaithersburg, MD) containing $0.5 \%$ trypsin (Canadian Life Technologies, Burlington, ON) and 0.1\% DNase (Sigma, St. Louis, MO) for $20 \mathrm{~min}$ at $37^{\circ} \mathrm{C}$. Cells were passed through a sterile nylon mesh (pore diameter 72 $\mu \mathrm{m})$ to remove nondissociated tissue, and trypsin was neutralized by adding an equal volume of minimum essential medium (MEM; Life Technologies-BRL) supplemented with $10 \%$ horse serum (Life Technologies-BRL). The cell suspension was collected in a sterile tube and centrifuged for $10 \mathrm{~min}$ at $2000 \mathrm{rpm}$. Pellets were resuspended in culture medium (MEM supplemented with $33 \mathrm{~mm}$ glucose, $4 \mathrm{~mm}$ glutamine, $26 \mathrm{~mm}$ sodium bicarbonate, $1 \mathrm{IU} / \mathrm{ml}$ penicillin-streptomycin, and $10 \%$ horse serum). Cells were counted in a hemocytometer, seeded into plastic Petri dishes (100 mm; Corning, Corning, NY) at a density of $\sim 20,000 / \mathrm{cm}^{2}$, and incubated at $37^{\circ} \mathrm{C}$ under $95 \% \mathrm{O}_{2}$ and $5 \% \mathrm{CO}_{2}$. To eliminate oligodendrocytes, their precursors, and microglia, culture medium was changed as soon as astrocytes had attached to the bottom of the dish, $2 \mathrm{hr}$ after plating, and dishes were shaken vigorously before each medium change (every $2 \mathrm{~d}$ ).

In a previous report, the virtual absence of oligodendrocyte precursors $(<0.1 \%)$ and the total absence of neurons after this procedure were assessed using immunocytochemistry for specific markers (Pierret et al., 1998a). To further characterize astrocyte cultures, in the present experiments, one Petri dish in each of three different cell culture preparations for each sampled brain region was double labeled with a polyclonal antiserum directed against GFAP (1:2000; Dakopatts, Glostrup, Denmark) to identify astrocytes, and with a lectin from Bandeirea simplicifolia (Griffonia simplicifilia-1 Isolectin $\mathrm{B}_{4}$, peroxidase-labeled, \#L5391; Sigma) to identify microglia (Streit and Kreutzberg, 1987). Immunoreactivity for GFAP was revealed with a rhodamine-labeled anti-rabbit IgG goat antiserum (1:200; Jackson, West Grove, PA), whereas lectin labeling of microglia was visualized by peroxidase reaction in the presence of 3,3'-diaminobenzidine (DAB, 0.05\%; Sigma) and $0.005 \%$ hydrogen peroxide. In each culture dish, labeled astrocytes and microglia were photographed in the same six microscopic fields, changing illumination from fluorescence to bright field, and counted on these photographs. The total number of microglial cells in a Petri dish was then expressed in percentage of the total number of cells.

Single immunoperoxidase labeling was also performed on three other astrocyte preparations from each brain region with a monoclonal antiserum against myelin/oligodendrocyte specific protein (MAB328; Chemicon, Temecula, CA), which confirmed the absence of oligodendrocytes. Positive controls for microglial and oligodendrocyte labeling were performed by preparing cultures without vigorous dish-shaking steps that showed good staining of microglia and oligodendrocytes, confirming the sensitivity of their detection.

Implantation. Confluent astrocyte cultures (20-22 d in vitro) were labeled with Hoechst fluorescent vital dye solution (bis-benzimidetrihydroclorate, Sigma; $10 \mu \mathrm{g} / \mathrm{ml}$ ) (Baron-Van Evercooren et al., 1992) at $37^{\circ} \mathrm{C}$ for $2 \mathrm{hr}$, rinsed three times in MEM, removed from the dish with trypsin-EDTA without $\mathrm{Ca}^{2+}$ and $\mathrm{Mg}^{2+}$ (Life Technologies), and suspended in MEM supplemented with $10 \%$ horse serum. After centrifugation $(10 \mathrm{~min}, 1700 \mathrm{rpm})$, the pellet was resuspended in the implantation medium (MEM supplemented with $0.22 \mathrm{gm} / 1$ sodium bicarbonate and $25 \mathrm{~mm}$ HEPES; Life Technologies) and centrifuged again, before mixing with fetal VM or striatal tissue.

For each session of implantation, VM was dissected from two litters of E15 rat fetuses (24-30 fetuses; crown-rump length $11 \mathrm{~mm}$ ). Only the rostral half of the VM was taken, to avoid including donor 5-HT neurons in the transplants. For striatal grafts, tissue from the lateral ganglionic 


\begin{tabular}{|c|c|c|c|c|c|c|c|c|c|c|c|c|c|c|}
\hline \multirow{2}{*}{$\begin{array}{l}\text { Survival time } \\
\text { Types of implants }\end{array}$} & \multicolumn{4}{|c|}{$10 \mathrm{~d}(15-40 \mathrm{~d})$} & \multicolumn{4}{|c|}{2 months } & \multicolumn{6}{|c|}{4 months } \\
\hline & VM & $\mathrm{Avm} / \mathrm{VM}$ & Astr/VM & $\mathrm{Acx} / \mathrm{VM}$ & $\mathrm{VM}$ & $\mathrm{Avm} / \mathrm{VM}$ & Astr/VM & $\mathrm{Acx} / \mathrm{VM}$ & VM & Avm/VM & Astr/VM & $\mathrm{Acx} / \mathrm{VM}$ & STR & Avm/STR \\
\hline Hoechst fluorescence & & 7 & 4 & 5 & & 10 & 17 & 18 & & & & & & \\
\hline \multicolumn{15}{|l|}{ Immunohistochemistry } \\
\hline GFAP & 3 & 7 & 5 & 3 & 1 & & 1 & & 1 & 2 & & 1 & 6 & 6 \\
\hline CSPG & 3 & $8(5)$ & 5 & $4(7)$ & 1 & & 1 & & & & & & & \\
\hline S- $100 \beta$ & 1 & & 1 & & & & & & 1 & 2 & & 1 & & \\
\hline Vimentin & & & & & & & & & 2 & 3 & & & & \\
\hline TH & 5 & $7(7)$ & 7 & $5(9)$ & & & & & & & & & & \\
\hline DARPP-32 & & & & & & & & & 1 & 2 & 1 & 1 & 6 & 6 \\
\hline 5-HT & 5 & $8(5)$ & 7 & $6(7)$ & 11 & 10 & 18 & 11 & & & & & 6 & 6 \\
\hline GABA & & (2) & & (2) & & & & & & & & & & \\
\hline Autoradiography $\left[{ }^{3} \mathrm{H}\right] 5-\mathrm{HT}$ & & & & & 8 & 9 & 11 & 9 & & & & & & \\
\hline Biotinylated dextran & & & & & & & & & 2 & 5 & & 3 & & \\
\hline
\end{tabular}

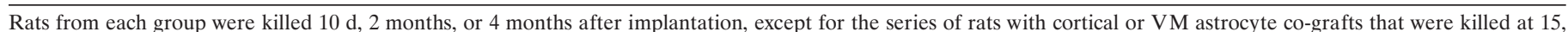

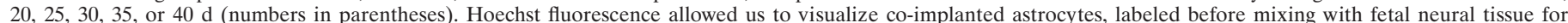

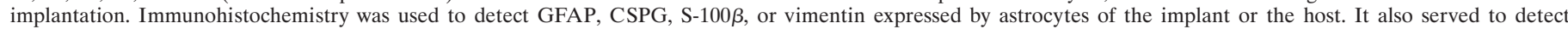

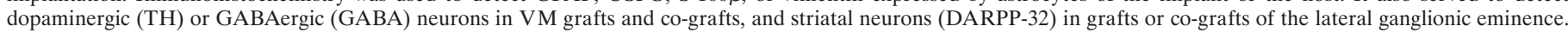

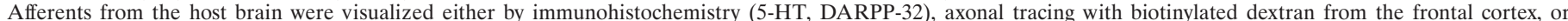

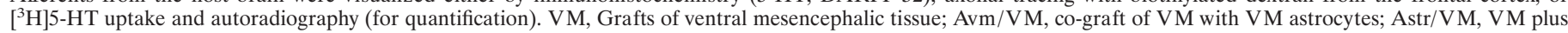

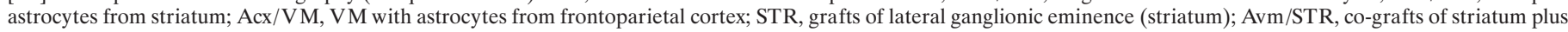
VM astrocytes.

eminence was dissected from two litters of E16 fetuses. Approximately one-third of the dissected tissue pieces $(\sim 8-10$ hemi-ventral midbrains or lateral ganglionic eminence) was kept for single, control grafts prepared according to the technique of Björklund et al. (1983). These and the other tissue pieces were incubated in parallel in implantation medium containing $0.5 \%$ trypsin and $0.1 \%$ DNase $\left(20 \mathrm{~min}\right.$ at $\left.37^{\circ} \mathrm{C}\right)$. For co-implantation experiments, astrocytes from one Petri dish $\left(\sim 2.5 \times 10^{6}\right.$ astrocytes) were added to the remaining tissue pieces ( $\sim 15-20$ ventral hemi-midbrains or lateral ganglionic eminence) before mechanical dissociation. The dissociated control VM or striatal suspension, as well as the mixtures of astrocytes and VM or striatal tissue, were centrifuged (3 min, $700 \mathrm{gm})$, and the pellets were resuspended in a small volume of implantation medium $(20-40 \mu \mathrm{l})$. Two stereotaxic injections of $2.5 \mu \mathrm{l}$ of the cell aggregate suspensions were injected with $10 \mu \mathrm{l}$ Hamilton microsyringes into the neostriatum of adult recipient rats at the following coordinates: $1 \mathrm{~mm}$ anterior to bregma, $3 \mathrm{~mm}$ to the right, and 5 and 4.1 $\mathrm{mm}$, respectively, below dura, with the tooth bar set at 0 . In each session, two rats received control single grafts and five others received co-grafts with astrocytes.

Histofluorescence and immunohistochemical characterization of the grafts. Several procedures were used to characterize the implants at different time points after implantation, as summarized in Table 1. For each surgery session, one control VM-grafted rat and two co-grafted rats were perfused $10 \mathrm{~d}$ later for immunohistochemistry to characterize the grafts, in particular the distribution of co-grafted astrocytes labeled with Hoechst fluorescent dye, the host and donor astrocytes (GFAP, vimentin), chondroitin sulfate proteoglycans (CSPGs), and the absence of co-grafted 5-HT perikarya. Tyrosine hydroxylase (TH) and GABA immunohistochemistry served to assess qualitatively the number and distribution of dopaminergic and GABAergic neurons and processes in the implants. Other rats were perfused 2 or 4 months after implantation for the same purposes. In addition, rats perfused at 4 months had received injections of biotinylated dextran at multiple sites into the frontal cortex, $15 \mathrm{~d}$ before, to label host cortical projections in the grafts by anterograde transport. In the same rats, dopamine- and adenosine-regulated phosphoprotein-32 (DARPP-32) immunohistochemistry on other series of sections allowed us to examine the presence of host striatum-derived afferents in the implants. Last, one series of VM grafts with co-grafted $\mathrm{VM}$ or cortical astrocytes was perfused after different survival times (15, $20,25,30,35$, and $40 \mathrm{~d}$ ) to assess the time course of TH and CSPG expression, as well as that of 5-HT axon ingrowth into the grafts.

For these experiments, the animals were deeply anesthetized with an overdose of sodium pentobarbital (Somnotol; MTC Pharmaceuticals, Cambridge, ON; $80 \mathrm{mg} / \mathrm{kg}$, i.p.) supplemented with methoxyflurane inhalation (Metofane, Janssen, Toronto, ON), and the brains were fixed by perfusion with $4 \%$ paraformaldehyde in $0.1 \mathrm{M}$ sodium-phosphate buffer ( $\mathrm{PB}, \mathrm{pH}$ 7.4), after a previous rinse of the vascular bed with sodium-PBS. For GABA immunohistochemistry, the rats (two with VM astrocyte co-grafts and two with cortical astrocyte co-grafts) were perfusion fixed with $3.5 \%$ glutaraldehyde. A thick coronal brain slice including neostriatum was further fixed in the same fixative, overnight at $4^{\circ} \mathrm{C}(1$ $\mathrm{hr}$ at room temperature for GABA), rinsed in PBS, and cut in four series of $50-\mu \mathrm{m}$-thick sections on a vibrating microtome.

The distribution of Hoechst-labeled astrocytes in the co-grafts was examined by fluorescence microscopy, in sections temporarily mounted in PBS on glass slides, under a coverslip. Hoechst fluorescence appeared blue with an excitation filter wavelength of $365 \mathrm{~nm}$ and a barrier filter wavelength of $420 \mathrm{~nm}$. These sections were recovered for further immunohistochemical staining.

Alternate series of sections were immunostained with a polyclonal antiserum against GFAP (1:2000; Dakopatts), a monoclonal antiserum against CSPGs (1:200; clone CS-56, Sigma), a polyclonal antiserum against 5-HT (1:5000; Diasorin, Stillwater, MN), and a polyclonal antiserum against TH (1:500; Pel-Freez Biologicals, Rogers, AR) for VM implants, or a primary monoclonal antibody against DARPP-32 (generous gift of Drs. E. L. Gustafson and P. Greengard, Rockefeller University) for striatal implants. For GABA, we used a monoclonal antibody anti-GABA (1:200, clone GB-69, Sigma). Immunoreactivity was revealed by the avidin-biotin-peroxidase method, with a biotinylated goat antiserum against rabbit IgGs (1:200; Sigma) or a biotinylated horse antiserum against mouse IgGs (1:100; Vector, Burlingame, CA) and with an avidin-biotin-peroxidase complex (Vectastain ABC kit; Vector). Peroxidase activity was revealed with DAB $(0.05 \%$; Sigma $)$ in the presence of $0.005 \%$ hydrogen peroxide.

Iontophoretic injections of biotin-dextran (10\% solution in saline; Molecular Probes, Eugene, OR) were made at six sites in the ipsilateral motor cortex, 4 months after implantation, under Hypnorm-Versed anesthesia. Injections were done using a glass micropipette (tip diameter $30 \mu \mathrm{m}$ ) connected to a current source (Midgard Precision Current Source, Stoelting, Wood Dale, IL) (5 $\mu$ A positive current, $7 \mathrm{sec}$ "on" and $7 \mathrm{sec}$ "off," for $15 \mathrm{~min}$ ) at the following coordinates: 2.7 and $3.2 \mathrm{~mm}$ anterior to bregma, 3, 2, and $1 \mathrm{~mm}$ to the right, and $1.5 \mathrm{~mm}$ below dura, with the tooth bar set at 0 . The micropipette was left in place for $1 \mathrm{~min}$ after each injection (Brandt and Apkarian, 1992). Fifteen days later, the brains were processed as above (immunohistochemistry). One series of sections was treated with the avidin-biotin-peroxidase complex (Vectastain $\mathrm{ABC}$ kit), and the peroxidase activity was revealed as above. Other sections from these animals were treated with monoclonal antibodies anti-DARPP-32, anti-S100 $\beta$ (Sigma; clone SH-B1; 1:1000) or anti-vimentin (Boehringer Mannheim, Mannheim, Germany; clone V9; $1: 100)$ and processed according to the peroxidase-anti-peroxidase (PAP) 
protocol, using a rabbit anti-mouse IgG antiserum (Dakopatts; 1:100) and mouse PAP (Dakopatts; 1:250).

$\left[{ }^{3} H\right] 5 H T$ uptake and autoradiography. Two months after implantation, 37 grafted animals, from 16 transplantation sessions $(4,5$ and 7 sessions, respectively, for VM, cortical, and striatal astrocyte co-grafts, with control VM grafts from 8 of these sessions) (Table 1) were processed for $\left[{ }^{3} \mathrm{H}\right] 5-\mathrm{HT}$ uptake and autoradiography according to a previously published procedure (Doucet and Descarries, 1993) described only briefly here.

The rats were perfused with ice-cold artificial CSF (CSF). After rapid dissection, 200- $\mu \mathrm{m}$-thick coronal slices of the striatum containing the graft were cut on a vibrating microtome. Three to four slices from each brain were preincubated for $15 \mathrm{~min}$ at $35^{\circ} \mathrm{C}$ in a solution of CSF containing an inhibitor of monoamine oxidase (pargyline, $0.1 \mathrm{~mm}$; Aldrich, Milwaukee, WI) and an inhibitor of monoamine uptake by dopaminergic terminals (benztropine-mesylate, $10 \mu \mathrm{M}$; Aldrich). The slices were incubated for $20 \mathrm{~min}$ at $35^{\circ} \mathrm{C}$ by adding [ $\left.{ }^{3} \mathrm{H}\right] 5-\mathrm{HT}(15-30 \mathrm{Ci} / \mathrm{mmol}$; Life Sciences, Boston, MA) to the preincubation solution. At the end of incubation, slices were flattened and fixed by immersion in sodium phosphate-buffered $3.5 \%$ glutaraldehyde $(0.1 \mathrm{M}, \mathrm{pH} 7.4)$ and by osmium vapors, dehydrated in increasing concentrations of ethanol, and embedded in epoxy resin (Epon 812, Mecalab, Montréal, Québec, Canada). Semithin sections ( $4 \mu \mathrm{m}$ thick) of the whole-brain slices were cut on a Polycut microtome (Reichert-Jung, Vienna, Austria), flattened onto glass slides, and autoradiographed by dipping in a liquefied nuclear emulsion (K-5, Ilford, Cheshire, UK). These autoradiographs were developed $21 \mathrm{~d}$ later in D-19.

Because this technique does not label dendrites or perikarya (Doucet and Descarries, 1993; Pierret et al., 1998a), the absence of donor 5-HT neurons in these grafts was assessed in adjacent $200-\mu \mathrm{m}$-thick slices fixed by immersion in $4 \%$ paraformaldehyde in $0.1 \mathrm{M} \mathrm{PB}$ containing $2.5 \%$ dimethylsulfoxide (Morin et al., 1997) for $5 \mathrm{~min}$ at $37^{\circ} \mathrm{C}$ and $4 \mathrm{hr}$ at $4^{\circ} \mathrm{C}$. These slices were first examined in fluorescence for Hoechst labeling of co-grafted astrocytes and then recovered and processed as above for immunoperoxidase with a polyclonal antiserum against 5-HT (1:5000; Diasorin). Because their thickness did not allow direct observation by light microscopy, they were embedded in paraffin and cut serially at a thickness of $7 \mu \mathrm{m}$ for observation.

Quantification and statistical analysis. All of the following measurements were performed on blind-coded sections, in random order, to avoid any bias attributable to camera drifting or the operator. The analyses were performed on a Macintosh Quadra computer using the public domain NIH Image Program (developed at the National Institutes of Health and available on the Internet at http://rsb.info.nih.gov/nihimage/). The computer was connected to a Leitz Orthoplan light microscope via a video camera (Panasonic WV-BD400, Matsushita, Japan).

Quantification of GFAP-immunoreactive processes was performed in five different sectors in each grafted brain: the core and the periphery of the implant; the host ipsilateral neostriatum, at 100 and $500 \mu \mathrm{m}$ from the implant; and the contralateral neostriatum, in a position symmetric to the graft. Immunostained elements were selected by gray scale adjustment, and their total area was measured in a window area of $10,000 \mu \mathrm{m}^{2}$. Four measurements were done in each sector. Results were expressed as the total area of the GFAP-immunostained elements over the reference area.

The intensity of CSPG immunostaining was assessed by measuring the "optical density" in a fixed area of $24,000 \mu \mathrm{m}^{2}$, using the "Mean Density" function of the NIH Image software. Four measurements were taken in the implant and four in the host neostriatum. Results were expressed as the ratio of density in the graft over the background density in the host neostriatum.

Serotonin axonal varicosities in autoradiographs were counted as described by Doucet and Descarries (1993). Labeled axonal varicosities were detected as small aggregates of silver grains over a background of diff use silver grains (see Fig. 12). The silver grain aggregates, visualized with a $16 \times$ PlanApo objective lens, were selected by gray scale adjustment, and the number of resulting binary image features was counted and expressed as number of labeled varicosities per square millimeter of tissue section. The counting window $\left(200,000 \mu \mathrm{m}^{2}\right)$ systematically scanned the complete area of the implants. Implants smaller than 180,000 $\mu \mathrm{m}^{2}$ were excluded from this analysis (11 of 48 grafts).

A one-way ANOVA and Fisher's post hoc test (Statview 4.01, Abacus Concepts, Berkeley, CA) were used for all comparisons among implants (single grafts and co-grafts).

Co-culture of dissociated dorsal raphe neurons on astrocytes from cortex or ventral midbrain. Complementary experiments were performed in vitro to examine the response of postnatal 5-HT neurons in the presence of astrocytes purified from either the cerebral cortex or ventral mesencephalon ( $n=3$ and 2, respectively). The neonatal [postnatal day (P) 0] astrocytes were plated as above, at a density of $\sim 80,000 / \mathrm{cm}^{2}$, in plastic Petri dishes $(20 \mathrm{~mm}$, Corning). At confluence, $20 \mathrm{~d}$ later, dissociated dorsal raphe neurons prepared from P10 rat pups according to the technique of Brewer (1997) were plated on top of the astrocytes at a density of $\sim 80,000 / \mathrm{cm}^{2}$. Cultures were maintained in MEM-glucose for a period of $7 \mathrm{~d}$. They were fixed with $4 \%$ paraformaldehyde in PBS containing $4 \%$ sucrose and then permeabilized with $0.3 \%$ Triton X-100 in PBS for $5 \mathrm{~min}$. The co-cultures were dually immunostained in sequence for GFAP and 5-HT, using the same polyclonal primary antibodies as above. They were visualized, respectively, with a fluorescein (FITC)-conjugated affinity-purified Fab fragment goat anti-rabbit IgG (1:50, H+L, Jackson ImmunoResearch Laboratories, West Grove, PA) and a rhodamine (TRICT)-conjugated affinity-purified goat anti-rabbit IgG (1:250, Jackson). Antibodies were dissolved in PBS containing 10\% BSA and $10 \%$ NGS, and extensive rinsing (several baths over $4 \mathrm{hr}$ ) was done between the incubation with the FITC-labeled Fab fragment and the incubation with the primary antibody anti-5-HT, to minimize cross reactions.

\section{RESULTS}

\section{Astrocytes represented $98 \%$ of the cells in the cultures from the three brain regions}

After 3 weeks in vitro, cell cultures double stained with Bandeirea simplicifolia B4 isolectin showed a uniform percentage of microglia $(2.13 \pm 0.37 \%$ for mesencephalic, $1.99 \pm 0.67 \%$ for cortical, and $1.71 \pm 0.23 \%$ for striatal astrocyte cultures) with no significant difference. This observation is important in the present context, because it has been shown that microglia may be four to eight times more numerous in mixed neuron-glia cultures of the mesencephalon compared with cortex (Kim et al., 2000). Oligodendrocytes were virtually absent from the present cultures, and neurons were previously shown to be absent also (Pierret et al., 1998a).

Astrocytes in culture showed subtle differences in shape, as described in a previous study (Pierret et al., 1998a). In particular, VM astrocytes tended to be more flattened than striatal or cortical ones (data not illustrated).

\section{Co-grafts contained more GFAP but other markers were very similar in all types of implants}

As reported previously (Krobert et al., 1997; Pierret et al., 1998a), a large proportion of the co-grafted astrocytes labeled with Hoechst fluorescent dye resided within the confines of the cografts, although many had migrated in the neighboring host striatum, in decreasing number with distance from the graft (Fig. 2). This distribution was comparable at $10 \mathrm{~d}$ for the three types of VM co-grafts and remained essentially the same after 2 months of survival.

Immunostaining for GFAP showed a higher number of reactive astrocytes within the implants than in the host striatum, as measured at $10 \mathrm{~d}$. The distribution of reactive astrocytes was comparable in all types of implants, with higher numbers at the graft/ host interface (Fig. 3A,B). Measurement of the relative area occupied by GFAP-immunoreactive processes was significantly higher in the core and at the border of the three types of co-grafts than in control VM grafts (see Fig. 4). There was no significant difference at the graft/host interface among co-grafts, but cografts with cortex-derived astrocytes had significantly higher values in their core than co-grafts with striatal or VM astrocytes.

GFAP immunostaining was still higher in the grafts than in the host striatum at 2 and 4 months after transplantation. The relative area covered by GFAP immunoreactive processes in the core of implants was not significantly different among animals perfused 

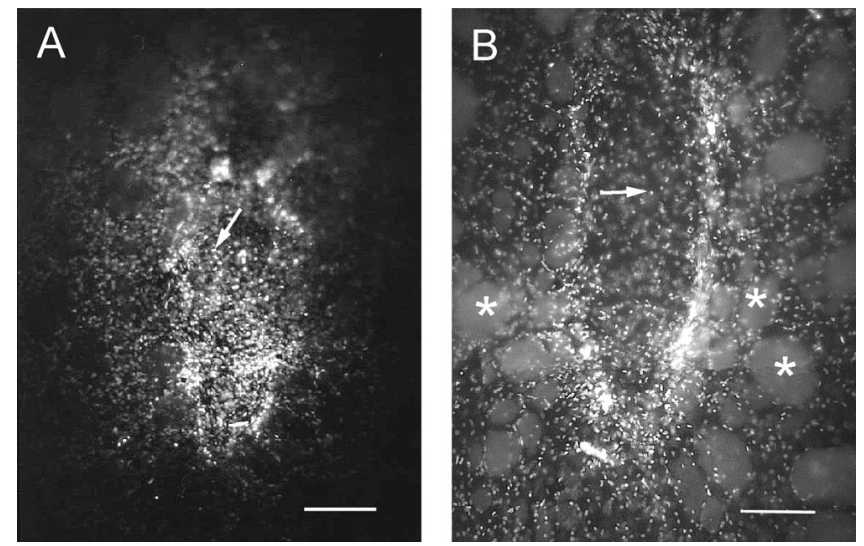

Figure 2. Co-grafted astrocytes labeled with Hoechst fluorescent dye reside in large numbers within the confines of the co-grafts. $A$, Co-graft of $\mathrm{VM}$ and VM astrocytes, $10 \mathrm{~d}$ after implantation. $B$, Co-graft of $\mathrm{VM}$ and striatal astrocytes, 2 months after implantation. Fascicles of the internal capsule, which aid in delineating host striatal tissue, are designated by asterisks in B. A large number of co-grafted astrocytes (arrows) are still residing in the implants, even after 2 months of survival. Comparable distributions were found in all implant groups. Scale bars, $400 \mu \mathrm{m}$.

at $10 \mathrm{~d}, 2$ months, or 4 months of survival. In the host ipsilateral striatum, measurements of GFAP-immunoreactive processes 500 $\mu \mathrm{m}$ lateral to the graft showed a significant reduction among $10 \mathrm{~d}$, 2 month $(p<0.05)$, and 4 month grafts $(p<0.001)$ (ANOVA and post hoc Fisher's test), whereas there was no such difference in the contralateral striatum.

Graft-related immunostaining for CSPGs was confined within the limits of the implants at all time points (Fig. $3 C-F$ ). In the host brain, there was staining in the subventricular zone and in the white matter, as in normal rats, but none associated with the implants. Densitometric measurement of CSPG immunoreactivity in the core of the grafts, at $10 \mathrm{~d}$, did not show any significant difference between control VM grafts and co-grafts or among the different types of co-grafts (see Fig. 5). Occasionally, CSPG immunoreactivity was slightly higher at the graft/host interface, but this was found with all four types of implants. Co-grafts in rats perfused at different survival times (VM vs cortical astrocyte co-grafts) showed a similar time course for CSPG expression in both types of co-grafts, with a clear downregulation occurring between 25 and $40 \mathrm{~d}$ (Fig. $3 E, F$ ). Two months after implantation, CSPG immunostaining was very weak, and it was then difficult to distinguish the graft with this staining.

Astrocytes immunostained for S- $100 \beta$ were ubiquitous in the brain of all implanted animals. Their distribution in relation to the implants was very similar to that of GFAP, except that S-100 $\beta$-immunostaining showed the perikarya more completely (Fig. 6). All implants were comparable with this staining.

Vimentin-positive astrocytes were still present in all implants, after 4 months of survival (see Fig. 7). These cells were distributed as patches of cell aggregates, confined to the grafts, and their distribution was comparable between implant types. They likely represented immature astrocytes, even 4 months after grafting, as described previously by Gates et al. (1996).

Tyrosine hydroxylase-immunostained dopaminergic neurons were uniformly and similarly distributed between the graft core and periphery in all types of implants, at $10 \mathrm{~d}$ after surgery. Again, all implants appeared similar at this time point. In animals with VM or cortical astrocyte co-grafts, killed at different survival times, the density and distribution of dopamine or GABA neu-
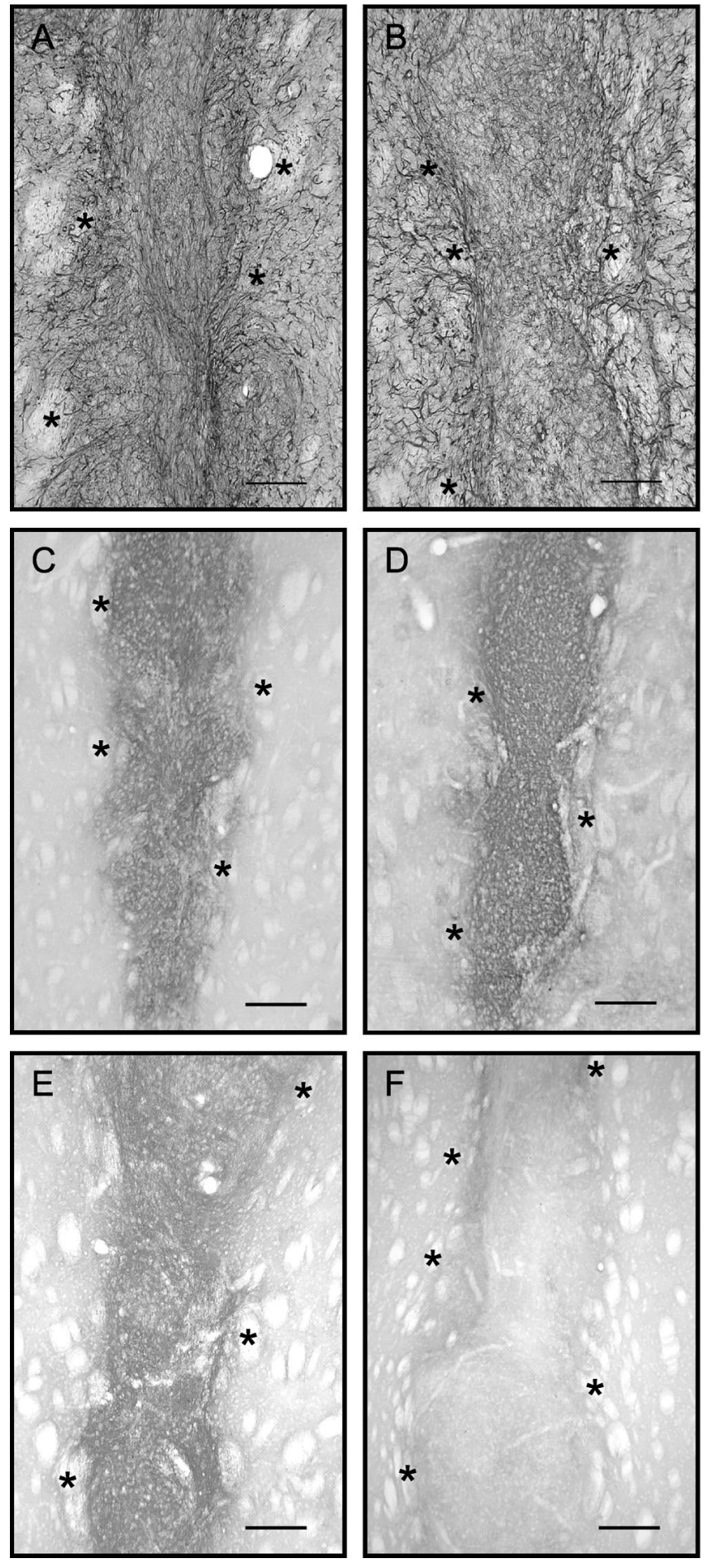

Figure 3. GFAP and CSPG immunoreactivities showed similar distributions in all types of implants. Co-grafts of $\mathrm{VM}$ with $\mathrm{VM}$ astrocytes $(A, C)$, VM with striatal astrocytes $(B, D)$, or VM with cortical astrocytes $(E, F)$ are shown as examples. $A$ and $B$ show sections of implants immunostained for GFAP; $C$ and $D$ display adjacent sections immunostained for CSPGs. No qualitative difference could be associated with a particular type of implant, although all types of co-grafts contained more GFAPimmunoreactive processes than VM single grafts, as shown in Figure 4.E and $F$ show sections from co-grafts of VM with cortical astrocytes, immunostained for CSPGs after $25 \mathrm{~d}(E$, same graft as in Fig. $9 D)$ or $40 \mathrm{~d}$ $(F)$ of survival. Asterisks indicate fascicles of the internal capsule, in host striatum. Scale bars: $A, B, 250 \mu \mathrm{m} ; C-F: 400 \mu \mathrm{m}$. 


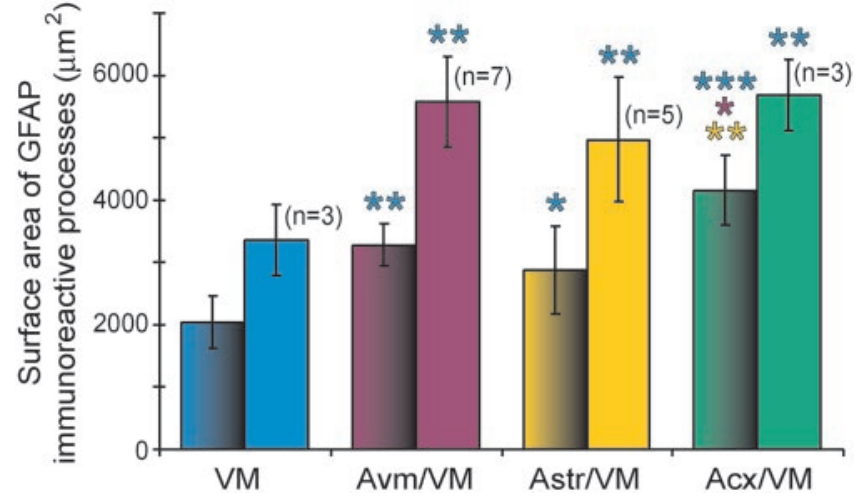

Figure 4. Relative surface area of GFAP-immunoreactive processes in the implants. The amount of astrocytes in the implants was estimated by measuring the total area of GFAP-immunoreactive processes, in the area covered by the measuring window. Measurements were done in the core of the implants (shaded columns) and at the graft-host border (uniform colors). Results are mean \pm SEM. One-way ANOVA and post hoc Fisher's test, $* p<0.05 ; * * p<0.01$; $* * * p<0.001$. All co-grafts showed higher values than single VM grafts, both in the core and at the graft-host border. Among co-grafts, the relative area of GFAP-immunoreactive processes was significantly higher in the core of those containing cortical astrocytes. $V M$, Control grafts of VM tissue; $A v m / V M$, co-graft of VM with VM astrocytes; $A s t r / V M, \mathrm{VM}$ plus astrocytes from striatum; $A c x /$ $V M, \mathrm{VM}$ with astrocytes from frontoparietal cortex. Color code: blue represents VM grafts; violet, VM with VM astrocytes; yellow, VM plus striatal astrocytes; and green, VM plus cortical astrocytes. The color of the asterisks refers to the group with which a significant difference was demonstrated. $n$ represents the number of implants in each group.

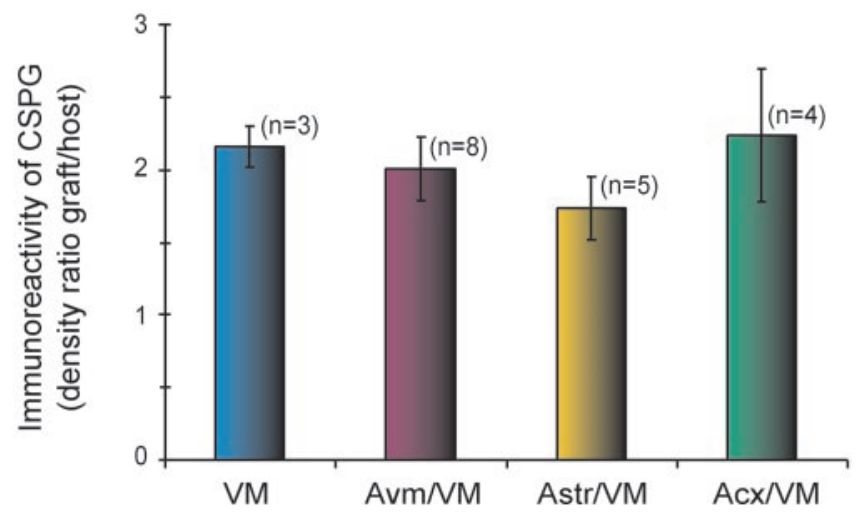

Figure 5. Intensity of CSPG immunoreactivity inside the implants. The density of CSPG immunostaining was measured as gray levels and expressed relative to the background density in the adjacent host neostriatum. There was no significant difference between groups. Statistical analyses, abbreviations, and color code as in Figure 4.

rons was also similar. Both types of grafts displayed dopamine neurons with well developed dendritic arbors and both contained a very high density of GABA-immunoreactive terminals (Fig. 8). Because host afferents into these grafts could not account for all these GABA terminals, we conclude that they originate from the grafts and that GABA neurons, as well as dopamine neurons, intrinsic to the graft developed similarly in the presence of both types of astrocytes.

The area of the implants was measured on autoradiographed sections (sections taken through the middle of the grafts). There was no significant difference between control grafts and co-grafts or between the different types of co-grafts, but there was a tendency for co-grafts with striatal $\left(0.50 \pm 0.10 \mathrm{~mm}^{2}\right)$ or cortical

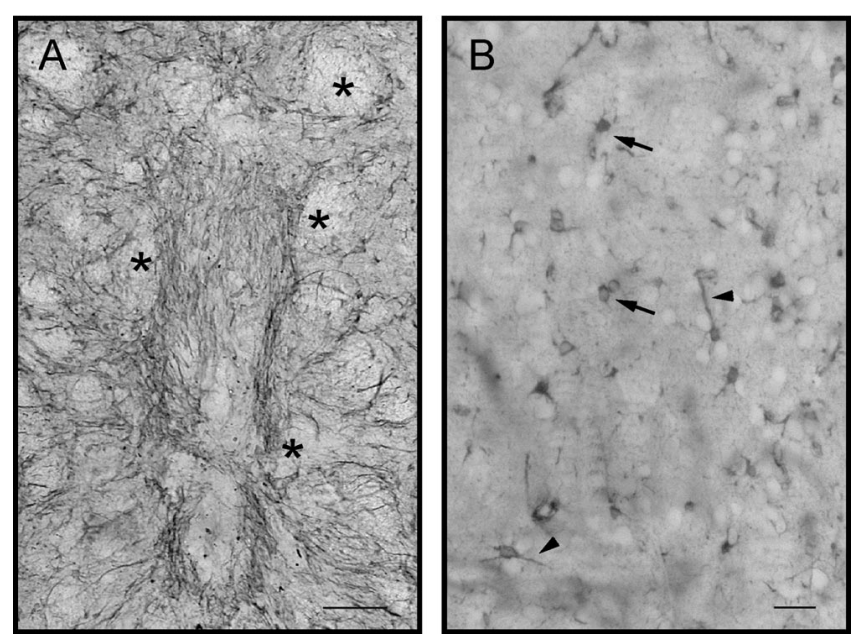

Figure 6. Astrocytes immunoreactive for S- $100 \beta$ had comparable distributions in all implanted rats. $A$ and $B$ show a co-graft of VM and VM astrocytes, $10 \mathrm{~d}$ after implantation, at low $(A)$ and high magnification $(B)$. Asterisks in $A$ indicate fascicles of the internal capsule. Arrows in $B$ point to labeled astrocytes. Note the similarity of the distribution of S- $100 \beta$ in $A$, with that of GFAP, in Figure $3 A$. The main difference between the two molecules is attributable to a better staining of host astrocytes and of astrocyte perikarya with $\mathrm{S}-100 \beta$ immunostain, as shown in $B$. No differences between types of implants were detected. Scale bars: $A, 250 \mu \mathrm{m} ; B$, $50 \mu \mathrm{m}$.

astrocytes $\left(0.44 \pm 0.05 \mathrm{~mm}^{2}\right)$ to be smaller than single grafts $\left(0.81 \pm 0.25 \mathrm{~mm}^{2}\right)$ and co-grafts with $\mathrm{VM}$ astrocytes $(0.68 \pm 0.16$ $\left.\mathrm{mm}^{2}\right)$. Considering the percentage of implants that were excluded from analysis because of their small size (none of the VM grafts or co-grafts with VM astrocytes vs $35 \%$ each for co-grafts with cortical or striatal astrocytes), the latter two were probably smaller, overall, as reported previously for co-grafts of VM with striatal astrocytes (Krobert et al., 1997; Pierret et al., 1998a).

\section{Serotonin immunohistochemistry suggested increased innervation of co-grafts containing cortical or striatal astrocytes}

Ten days after implantation, a few 5-HT-immunopositive fibers were visualized within all four types of implants (see Fig. 9A,B). Most of such fibers were in the periphery, but some were present well within the core of the implants. At this time point, there was no obvious difference in 5-HT innervation among the different types of implants. In the series of animals with VM or cortical co-grafts perfused at different survival times, it was observed that a difference in 5-HT innervation became apparent at $15 \mathrm{~d}$, with cortical astrocyte co-grafts containing more 5-HT axons than VM astrocyte co-grafts (see Fig. 9C,D). Two months after implantation, 5-HT-immunostained fibers also appeared more numerous in co-grafts containing cortical or striatal astrocytes than in VM grafts or co-grafts with VM astrocytes (data not shown).

Serotonin neuronal perikarya were not detected in any of the implants, confirming the host origin of the 5-HT-immunostained axons.

\section{Autoradiography demonstrated higher 5-HT innervation of co-grafts with cortical or striatal astrocytes}

After $\left[{ }^{3} \mathrm{H}\right] 5$-HT uptake labeling, light microscope autoradiographs displayed numerous silver grain clusters over a diff use background of individual silver grains (see Fig. 10). It was shown 

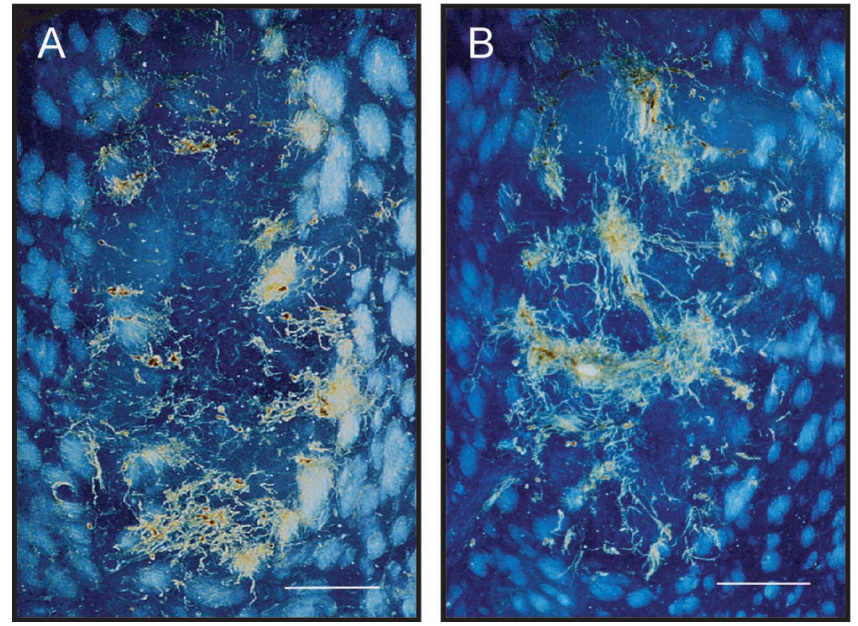

Figure 7. The distribution and amounts of vimentin-immunoreactive cells were comparable in all groups of implants. $A$, Single VM graft. $B$, Co-graft of VM and VM astrocytes. Both sections were immunostained for vimentin, after 4 months of survival. Vimentin-immunoreactive astrocytes were found in clusters, suggesting persistence of astrocytes in an immature state. These cells were presumably derived from the fetal VM tissue rather than from the co-grafted cultured astrocytes, because they were found in comparable amounts in single VM grafts and in co-grafts. Scale bars, $250 \mu \mathrm{m}$.

previously that such silver grain clusters represent 5-HT axonal varicosities (Doucet and Descarries, 1993).

Counts of silver grain clusters in the control VM grafts and in the three types of co-grafts revealed significant differences in 5-HT innervation among the groups of implants (Fig. 11). Cografts with cortical or striatal astrocytes contained four and three times, respectively, more 5-HT axonal varicosities than VM grafts or co-grafts with VM astrocytes. The difference between co-grafts with cortical versus striatal astrocytes was also statistically significant. Within each control or experimental group of implants, there was no significant difference in the density of
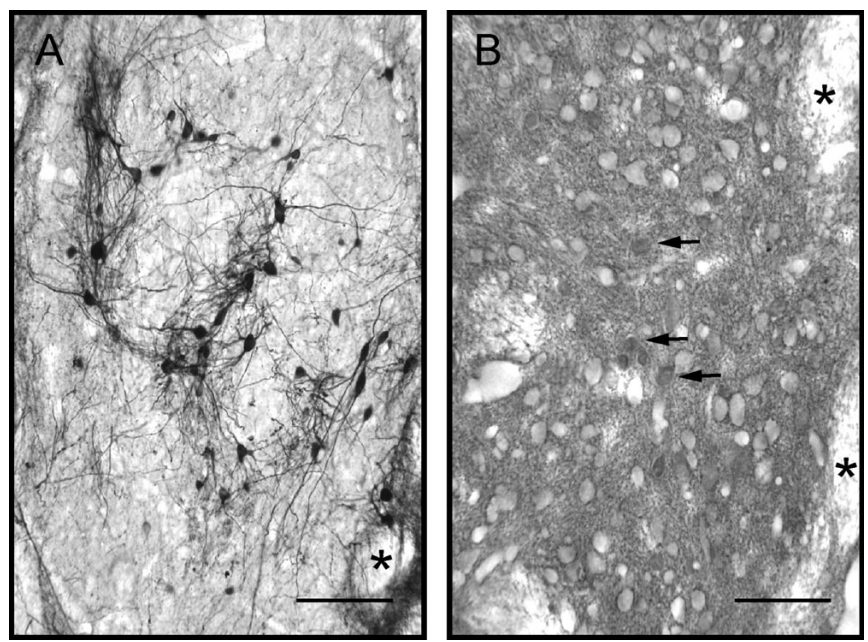

Figure 8. Distribution and maturation of dopamine and GABA neurons were similar in all types of implants. Exemplified is a co-graft of VM and $\mathrm{VM}$ astrocytes in adjacent sections immunostained for tyrosine hydroxylase $(A)$ or GABA $(B)$, after $40 \mathrm{~d}$ of survival. Arrows indicate immunolabeled GABA perikarya. Asterisks point to myelinated axon fascicles of the internal capsule in the host neostriatum. All types of implants had similar aspects with these immunostains. Scale bars, $50 \mu \mathrm{m}$.
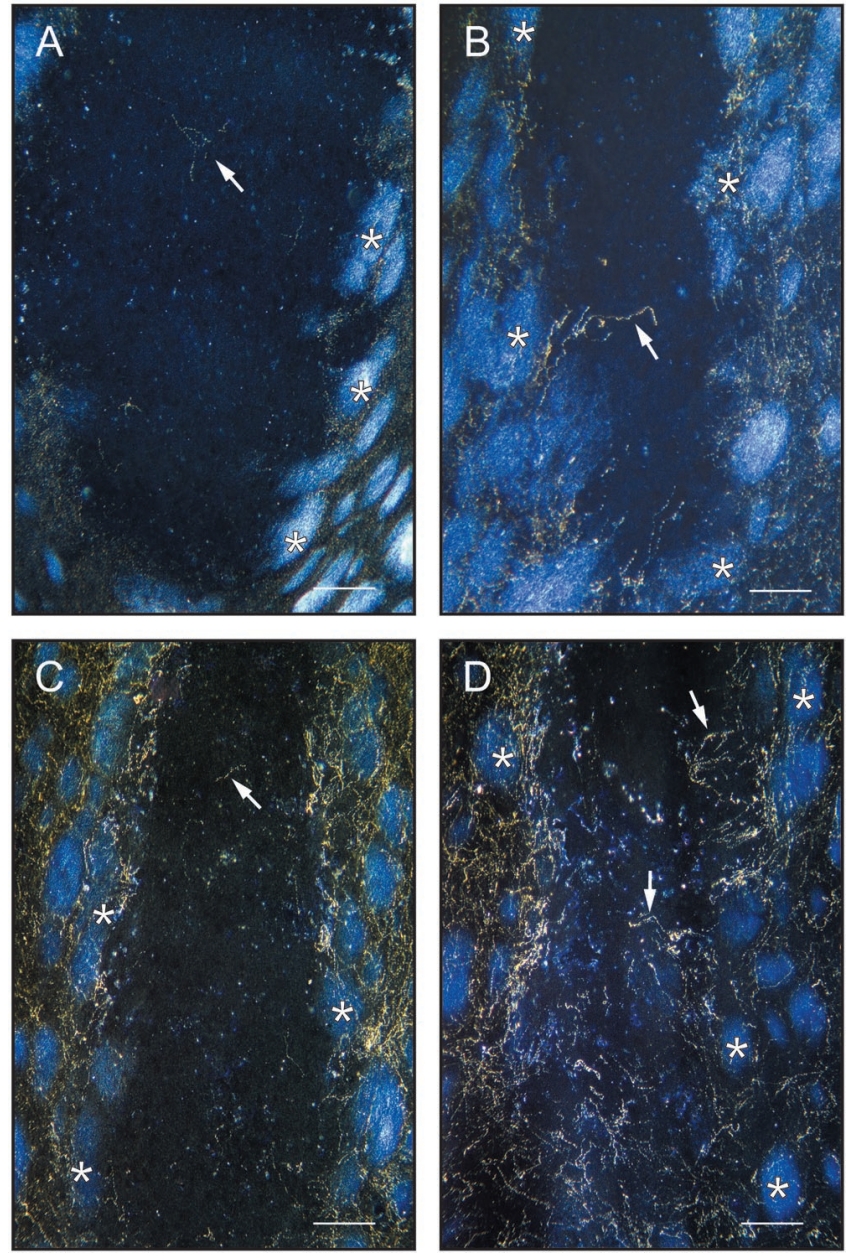

Figure 9. The number of 5-HT immunoreactive axons is higher in co-grafts with cortical (or striatal) astrocytes and increases with survival time. $A$, Control VM graft, $10 \mathrm{~d}$ after implantation; $B$, co-graft with cortical astrocytes, $10 \mathrm{~d}$ after implantation; $C$, co-graft with VM astrocytes, $25 \mathrm{~d}$ after implantation; $D$, co-graft with cortical astrocytes, $25 \mathrm{~d}$ after implantation (same graft as in Fig. $3 E$ ). Note the higher number of immunoreactive axons inside the implant in $D$ than in the one in $C$. Note also the absence of 5-HT-immunoreactive perikarya, demonstrating that the immunostained axons take origin in the host brain. Pictures were taken with dark-field illumination. The implants are easily distinguished from the host neostriatal tissue, which is recognized by the bright myelinated fascicles of the internal capsule. Arrows point to examples of immunolabeled axons. Scale bars, $250 \mu \mathrm{m}$.

5-HT innervation between animals implanted in the different surgery sessions.

Immunohistochemistry performed on sections adjacent to the incubated slices showed no 5-HT neuronal perikarya in these grafts.

\section{Ventral mesencephalic astrocytes had no effect on 5- HT innervation of striatal tissue grafts}

Transplants of fetal lateral ganglionic eminence with or without co-implanted VM astrocytes were prepared to test the possibility that VM astrocytes are inhibitory to 5-HT axon growth. These implants, examined by 5-HT immunohistochemistry after 4 months of survival, were all very well innervated by host-derived 5-HT axons (data not shown). Therefore, VM astrocytes show no inhibitory effect on the 5-HT innervation of the grafts. 

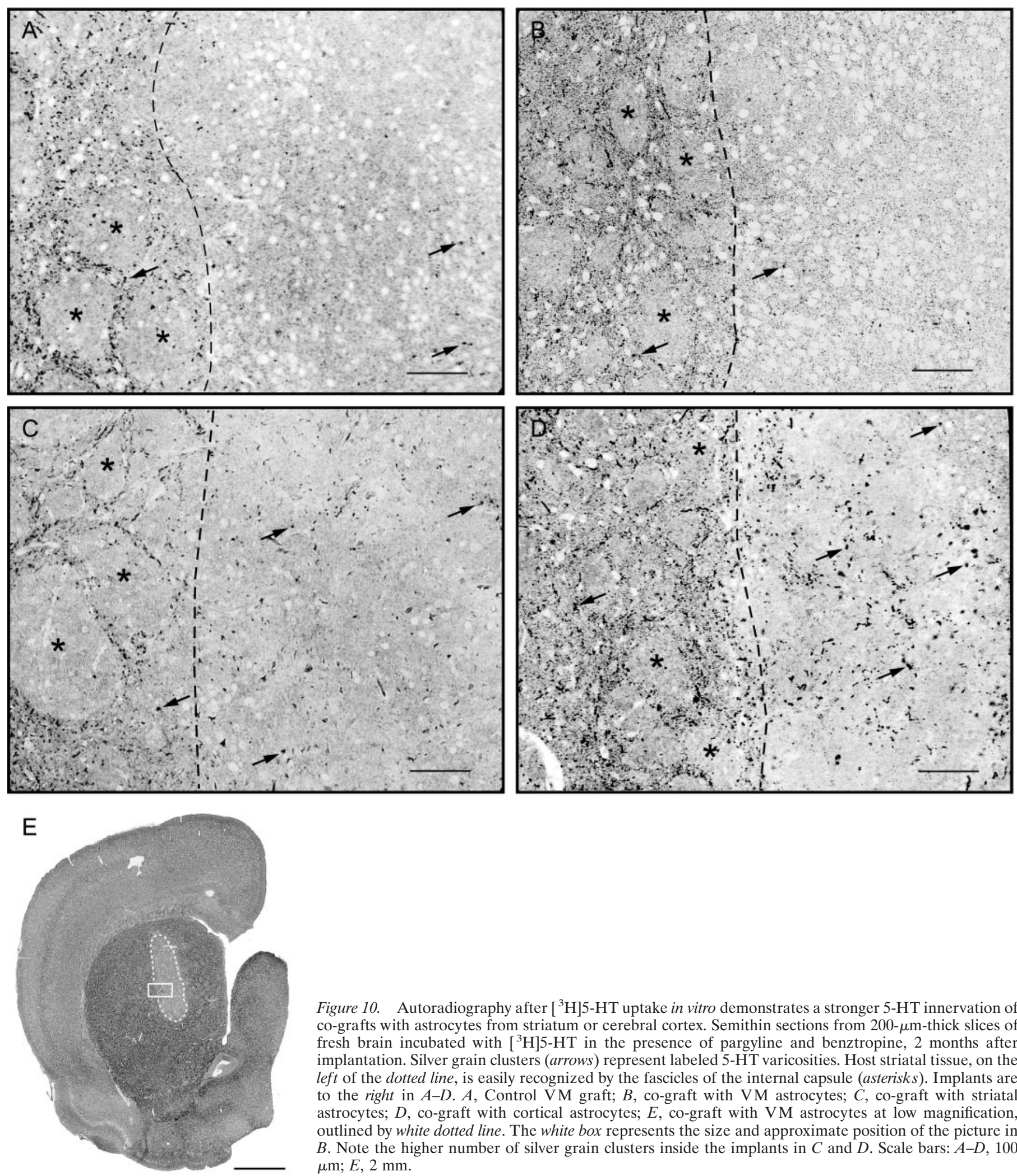

Figure 10. Autoradiography after $\left[{ }^{3} \mathrm{H}\right] 5$-HT uptake in vitro demonstrates a stronger 5-HT innervation of co-grafts with astrocytes from striatum or cerebral cortex. Semithin sections from $200-\mu \mathrm{m}$-thick slices of fresh brain incubated with $\left[{ }^{3} \mathrm{H}\right] 5-\mathrm{HT}$ in the presence of pargyline and benztropine, 2 months after implantation. Silver grain clusters (arrows) represent labeled 5-HT varicosities. Host striatal tissue, on the left of the dotted line, is easily recognized by the fascicles of the internal capsule (asterisks). Implants are to the right in $A-D$. $A$, Control VM graft; $B$, co-graft with VM astrocytes; $C$, co-graft with striatal astrocytes; $D$, co-graft with cortical astrocytes; $E$, co-graft with VM astrocytes at low magnification, outlined by white dotted line. The white box represents the size and approximate position of the picture in $B$. Note the higher number of silver grain clusters inside the implants in $C$ and $D$. Scale bars: $A-D, 100$ $\mu \mathrm{m} ; E, 2 \mathrm{~mm}$.

\section{Host cortical and striatal projections were very similar into the different types of implants}

Axonal tracing with biotinylated dextran injected into the frontal cortex revealed many labeled axons inside all implants (Fig. 12A). As described previously for single VM grafts (Doucet et al., 1989), axons of cortical origin were present in all areas of the grafts (Fig. 12B). The innervation of single VM grafts and of co-grafts with cortical or VM astrocytes appeared very similar in amounts and morphology.

DARPP-32-immunopositive axons were present essentially in the periphery of the implants, penetrating for $\sim 50-100 \mu \mathrm{m}$ (Fig. 12C), although some could be traced to the graft core, as 


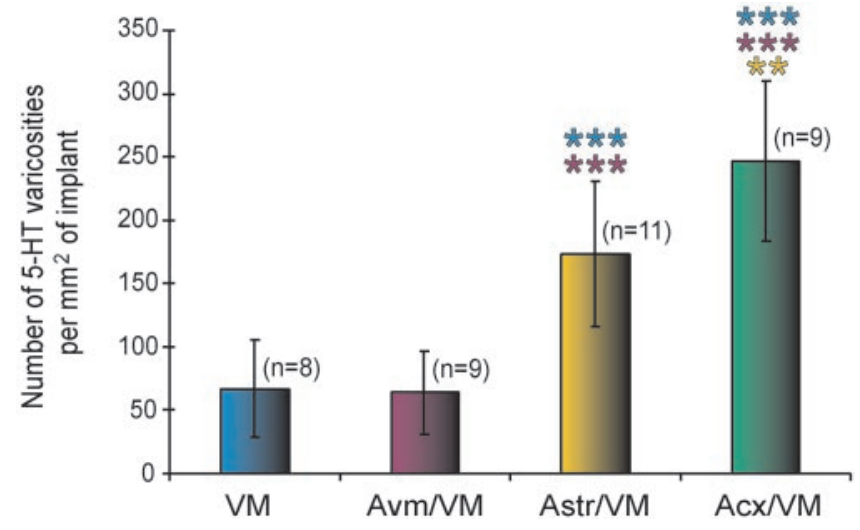

Figure 11. Relative numbers of 5-HT varicosities in the different types of implants. All silver grain clusters present in the implant were counted by image analysis in every section and expressed as numbers of 5-HT varicosities per square millimeter of implant area. Statistical analyses, abbreviations, and color code as in Figure 4.

described previously for single VM grafts (Doucet et al., 1989; Chkirate et al., 1993). Again, the picture was very similar for VM graft and co-grafts with cortical, mesencephalic, or striatal astrocytes.

\section{Serotonin neurons grew similarly well on VM and cortical astrocyte cultures}

Postnatal 5-HT neurons taken from P10 dorsal raphe and plated onto confluent VM or cortical astrocyte cultures survived similarly and displayed comparably extensive neurite branching (Fig. 13). This expected result exemplifies the necessity for in vivo experi- ments to examine axon guidance. Indeed, 5-HT neurons of the dorsal raphe project to most brain regions and are highly collateralized. The different paths followed by collaterals of a single neuron are presumably guided by different molecular cues. The response of axons to guidance cues also changes along the way toward their targets, as shown by Stein and Tessier-Lavigne (2001), for example.

\section{DISCUSSION}

The present study demonstrates that astrocytes from the neonatal cerebral cortex or striatum increase the 5-HT innervation of VM grafts implanted into the neostriatum of adult rats, whereas VM astrocytes have no such effect. It also shows that this increase cannot be attributed to a reduction of the glial scar or of the expression of CSPGs in the graft. Axonal tracing with biotinylated dextran and DARPP-32 immunohistochemistry revealed no obvious difference between implants in their host-derived cortical or striatal innervations. The simplest and most likely hypothesis to explain the increase in 5-HT innervation is that cortical and striatal astrocytes express a molecule or molecules that attract 5-HT axons present in the neighboring host neostriatum.

\section{Cortical and striatal astrocytes increase host 5-HT innervation of VM grafts}

The quantitative data on the 5-HT innervations of the implants were obtained using a technique developed specifically to count monoamine axonal varicosities in rat brain (Soghomonian et al., 1987; Doucet et al., 1988; Doucet and Descarries, 1993). For 5-HT axonal varicosities, this technique is based on the uptake of $\left[{ }^{3} \mathrm{H}\right] 5-\mathrm{HT}$ in the presence of an inhibitor of monoamine uptake by dopamine neurons (benztropine) and an inhibitor of mono-
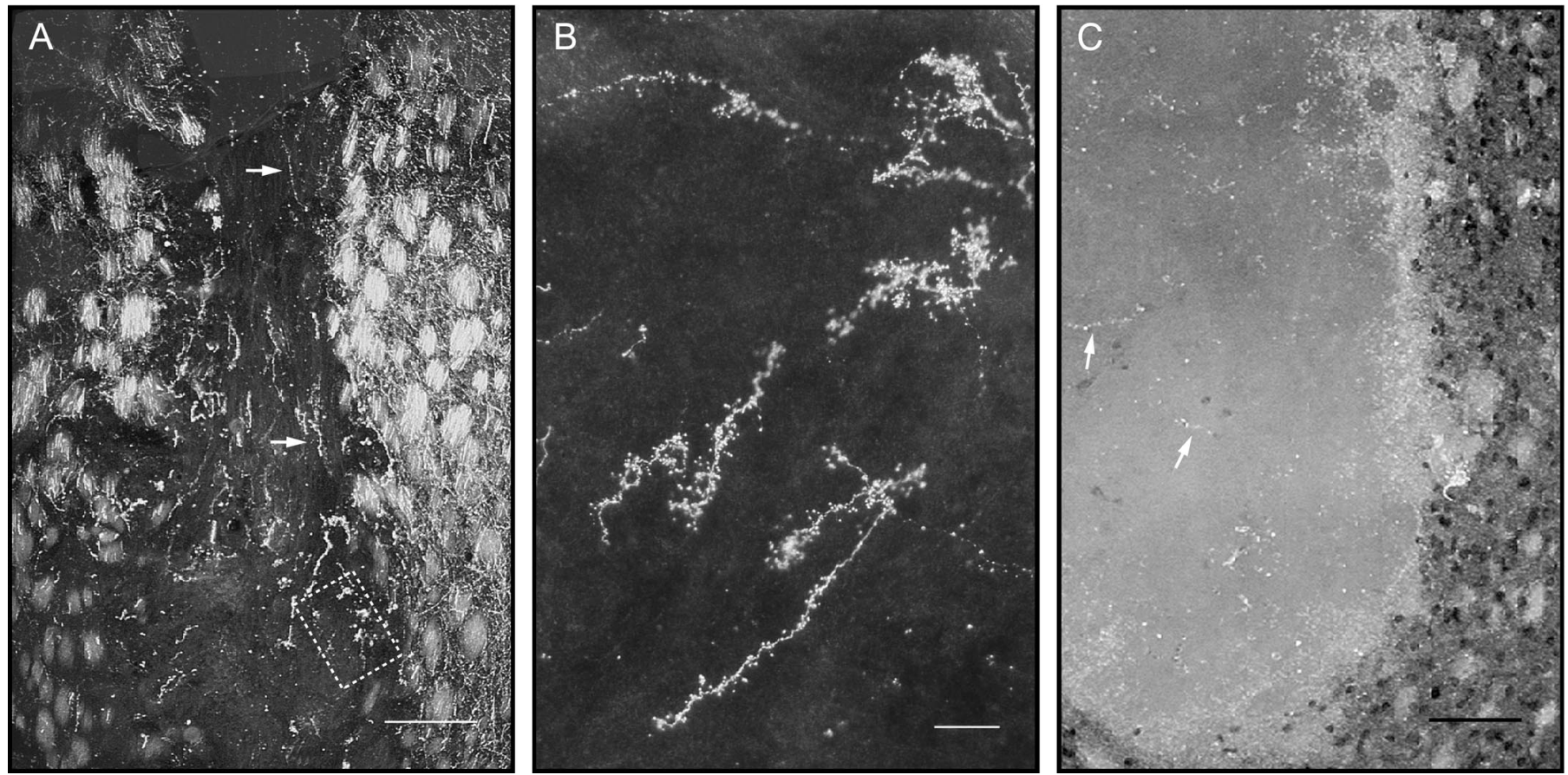

Figure 12. Axonal projections from the host cerebral cortex and striatum are not affected by co-grafted astrocytes. $A$, Cortical projections were labeled by anterograde axonal transport of biotinylated dextran after multiple (6) iontophoretic injections in the frontoparietal cortex, 2 weeks before animals were killed, and 4 months after implantation. Note the presence of labeled axons in the host internal capsule and in all regions of this co-graft with VM astrocytes. $B$, Higher magnification of box in $A$, showing the typical tortuous shape of cortical axons in the implant. $C$, Section of a control VM graft immunostained for DARPP-32, showing immunoreactive axons coming from the host striatum, mainly at the periphery, but with some well in the core of the implant (arrowheads). Labeled perikarya of the host striatum appear as black profiles, on the right of the picture. Pictures were taken with dark-field illumination. All implants looked very similar in the amounts of host cortical and striatal afferents. Scale bars: $A, 400 \mu \mathrm{m} ; B, 100 \mu \mathrm{m} ; C, 200 \mu \mathrm{m}$. 

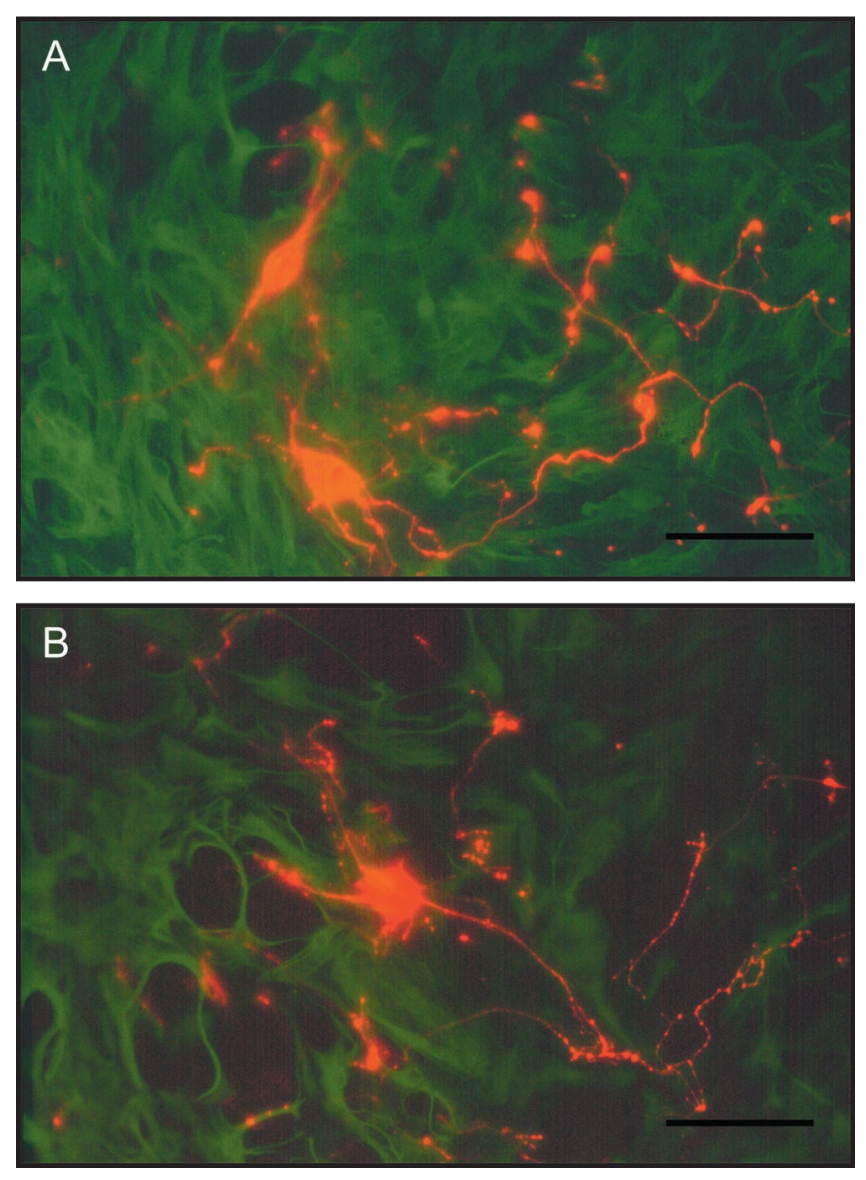

Figure 13. P10 dorsal raphe serotonin neurons in co-culture with astrocytes from the neonatal VM $(A)$ or cerebral cortex $(B)$ after $7 \mathrm{~d}$ in vitro. Astrocytes are immunostained for GFAP (green, fluorescein or FITC), and 5-HT neurons are immunostained for 5-HT (red, rhodamine or TRICT). Serotonin neurons survived and developed neurites equally well in the presence of both types of astrocytes. Scale bars, $50 \mu \mathrm{m}$.

amine oxidase (pargyline). As discussed elsewhere (Doucet and Descarries, 1993), this approach offers several advantages over immunohistochemistry for the quantification of monoamine terminals. In summary, labeling conditions are controlled more rigorously, reducing variations attributable to technical factors, whereas counting silver grain clusters in a thin nuclear emulsion is fairly easy to perform by image analysis.

The fact that no 5-HT neurons were detected in any of the implants confirms that no 5-HT neurons were included in the tissue pieces during dissection of the fetuses. Therefore, we can conclude that the quantitative differences in 5-HT innervation represent differences in ingrowth of 5-HT axons from the host brain. The number of 5-HT varicosities counted in co-grafts with cortical astrocytes may be estimated at $2.4 \times 10^{5} / \mathrm{mm}^{3}$ (taking into account incomplete detection of tritium-labeled varicosities in 4- $\mu \mathrm{m}$-thick sections, autoradiographic exposure time, and size of the varicosities). This figure represents 3 or $15 \%$, respectively, of the numbers reported previously for the rat substantia nigra (Moukhles et al., 1997) or neostriatum (Soghomonian et al., 1987). These values are consistent with the idea of a reduced growth capacity intrinsic to adult neurons. Indeed, the 5-HT innervation of VM or striatal grafts in adult recipients represents only $25 \%$ of that of similar grafts in neonatal recipients (Pierret et al., 1998b).

\section{The differences in 5-HT innervation were not caused by an indirect effect of co-grafted astrocytes on the glial scar}

After CNS damage or neural transplantation, reactive astrocytes form a glial scar and upregulate their expression of extracellular matrix molecules, such as CSPGs, which may be inhibitory to axonal growth (McKeon et al., 1991; Gates et al., 1996; Haas et al., 1999). For control VM grafts, the present observations on the distribution and time course of GFAP and CSPG expression are entirely consistent with the report of Gates et al. (1996), who also demonstrated that most of the CSPGs is produced by the grafted tissue. All implants contained reactive astrocytes and a glial scar at the graft/host interface, as reported by Krobert et al. (1997). Measurements of GFAP-immunoreactive processes showed an increase in all types of co-grafts, compared with single VM grafts, consistent with an enrichment of co-grafts with astrocytes expressing GFAP. Co-grafts with VM astrocytes contained similar amounts of GFAP-immunoreactive processes at the graft/host interface as co-grafts with cortical or striatal astrocytes. Thus, differences in the glial scar cannot account for the higher innervation of the latter two types of co-grafts by host 5-HT axons. The only difference among co-grafts concerned the higher values for the total area of GFAP-positive processes in the core of co-grafts containing cortical astrocytes, but this difference does not support the hypothesis of an impediment of axon ingrowth by a glial scar, because these co-grafts were the most densely 5-HT innervated.

The absence of difference in the immunoreactivity for CSPGs also disqualifies these molecules as being responsible for differences in the ingrowth of host 5-HT fibers into the co-grafts. Moreover, CSPG immunoreactivity had almost disappeared after 40 d of survival, as also described by Gates et al. (1996). Interestingly, immunoreactive 5-HT axons were already more numerous in the implants containing cortical astrocytes, compared with those with VM astrocytes, $15 \mathrm{~d}$ after implantation. Therefore, 5-HT axons began to grow into the implants before the downregulation of CSPG expression occurred (after $25 \mathrm{~d}$ ), suggesting that CSPGs were not inhibitory to host 5-HT fiber ingrowth.

The results with GFAP and CSPGs suggested that co-grafting of cultured astrocytes had no influence on the glial scar or on the production of CSPGs in the implanted tissue. Their impact on the expression of tenascin, another axon growth inhibitory molecule, was not examined, but the results of Gates et al. (1996) demonstrated that the host astrocytic and extracellular matrix molecule (CSPG and tenascin) response was the same for grafts of VM or of lateral ganglionic eminence. Moreover, TH and GABA neuronal populations were comparable between co-grafts. It seems likely, thus, that an attractive influence of co-grafted cortical and striatal astrocytes was responsible for their growthpromoting effect on 5-HT axons. This attractive influence might be attributable to a more "immature" state of neonatal telencephalic astrocytes in comparison with VM astrocytes (McKeon et al., 1991). However, one would expect a general axon growthpromoting action of immature astrocytes that would act uniformly on any type of host axons, which is not supported by the observation that cortical or striatal projections into the implants were not affected by co-grafted astrocytes. Indeed, projections from the host frontal cortex were already relatively prof use in control VM grafts, as reported previously (Doucet et al., 1989), and this did not change with co-grafted astrocytes, whereas those from neostriatum, labeled by DARPP-32 immunostaining, remained confined mainly to the periphery of all implants, with only occa- 
sional axons in the core of the implants, as also reported before for single VM grafts (Doucet et al., 1989; Chkirate et al., 1993).

We conclude from these results that the effect of co-grafted cortical and striatal astrocytes is specific for 5-HT axons, at least among the examined host brain regions, and therefore likely reflect production of specific growth guidance, rather than generally growth promoting, molecules. This effect is apparently specific for 5-HT axons innervating the striatum, because VM and cortical astrocytes in culture had similar effects in co-culture on postnatal 5-HT neurons of the dorsal raphe.

\section{Candidate molecules}

We had shown previously that host 5-HT axons of the neostriatum had a preference for grafts of cortical or striatal tissue (Pierret et al., 1998b) (A. Petit, N. Quenneville, P. Pierret, and G. Doucet, unpublished observations). The complexity of these tissues precluded attempts to identify responsible molecules. The present results simplify the situation because relatively pure populations of cells are shown to have similar effects. We propose that astrocytes from the cerebral cortex and striatum express molecules that have attractive effects on the 5-HT axons that normally innervate the telencephalon. The few 5-HT axons that penetrated control VM grafts might be from neurons sending collaterals to both the striatum and substantia nigra (Van der Kooy and Hattori, 1980). It remains to be seen whether the attractive molecules produced by cortical and striatal astrocytes are the same.

Many types of molecules with axon guidance properties, including cell or matrix adhesion molecules, as well as trophic and tropic factors, can be expressed by astrocytes, at least in vitro (Vickland and Silver, 1996). Some neurotrophic factors that may be expressed by astrocytes have already been shown to exert an effect on the growth of 5-HT axons. These include S-100 $\beta$ (Azmitia et al., 1990; Whitaker-Azmitia et al., 1990), brain-derived neurotrophic factor (Mamounas et al., 1995, 2000), NT-3 (Xu et al., 1995), and glial cell line-derived neurotrophic factor (Beck et al., 1996). Because all implants displayed similar distributions of S- $100 \beta$-positive astrocytes, this trophic factor cannot account for the differences in 5-HT innervation.

Other guidance molecules are likely to be discovered with the current advances in genomics and proteomics. One advantage of the present in vivo model is that the astrocytes grown in culture appear to maintain the regional phenotype that influences 5-HT axons. It should therefore be feasible to compare gene expression between the three different populations of astrocytes to determine whether known or unknown genes are differentially expressed among them. The products of such genes could be tested for an effect on 5-HT axon outgrowth in culture. Their expression might then be blocked in cortical or striatal astrocytes before co-grafting with VM tissue to determine whether they have a differential effect on a specific population of 5-HT axons, i.e., those projecting to the neostriatum.

\section{REFERENCES}

Altman J, Bayer SA (1995) Atlas of prenatal rat brain development. Boca Raton, FL: CRC.

Azmitia EC, Perlow MJ, Brennan MJ, Lauder JM (1981) Fetal raphe and hippocampal transplants into adult and aged C57BL/6N mice: a preliminary immunocytochemical study. Brain Res Bull 7:703-710.

Azmitia EC, Dolan K, Whitaker-Azmitia PM (1990) S-100 B but not NGF, EGF, insulin or calmodulin is a CNS serotoninergic growth factor. Brain Res 516:354-356.

Baron-Van Evercooren A, Clerin-Duhamel E, Lapie P, Gansmuller A, Lachapelle F, Gumpel M (1992) The fate of Schwann cells transplanted in the brain during development. Dev Neurosci 14:73-84.

Beck KD, Irwin I, Valverde J, Brennan TJ, Langston JW, Hefti F (1996)
GDNF induces a dystonia-like state in neonatal rats and stimulates dopamine and serotonin synthesis. Neuron 16:665-673.

Björklund A, Stenevi U, Schmidt RH, Dunnett SB, Gage FH (1983) Intracerebral grafting of neuronal cell suspensions. II. Survival and growth of nigral cell suspensions implanted in different brain sites. Acta Physiol Scand [Suppl] 522:9-18.

Brandt HM, Apkarian VA (1992) Biotin-dextran: a sensitive anterograde tracer for neuroanatomic studies in rat and monkey. J Neurosci Methods 45:35-40.

Brewer GJ (1997) Isolation and culture of adult rat hippocampal neurons. J Neurosci Methods 71:143-155.

Castro AJ, Tonder N, Sunde NA, Zimmer J (1988) Fetal neocortical transplants grafted to the cerebral cortex of newborn rats receive afferents from the basal forebrain, locus coeruleus and midline raphe. Exp Brain Res 69:613-622.

Chen MS, Huber AB, van der Haar ME, Frank M, Schnell L, Spillmann AA, Christ F, Schwab ME (2000) Nogo-A is a myelin-associated neurite outgrowth inhibitor and an antigen for monoclonal antibody IN-1. Nature 403:434-439.

Chkirate M, Vallée A, Doucet G (1993) Host striatal projections into fetal ventral mesencephalic tissue grafted to the striatum of immature or adult rat. Exp Brain Res 94:357-362.

Deacon TW, Pakzaban P, Burns LH, Dinsmore J, Isacson O (1994) Cytoarchitectonic development, axon-glia relationships, and long distance axon growth of porcine striatal xenografts in rats. Exp Neurol 130:151-167.

Denis-Donini S, Estenoz M (1988) Interneurons versus efferent neurons: heterogeneity in their neurite outgrowth response to glia from several brain regions. Dev Biol 130:237-249.

Denis-Donini S, Glowinski J, Prochiantz A (1984) Glial heterogeneity may define the three-dimensional shape of mouse mesencephalic dopaminergic neurones. Nature 307:641-643.

Doucet G, Descarries L (1993) Quantification of monoamine innervations by light microscopic autoradiography following tritiated monoamine uptake in brain slices. Neurosci Protoc 93-050-009-001-015.

Doucet G, Descarries L, Audet MA, Garcia S, Berger B (1988) Radioautographic method for quantifying regional monoamine innervations in the rat brain: application to the cerebral cortex. Brain Res 441:233-259.

Doucet G, Murata Y, Brundin P, Bosler O, Mons N, Geffard M, Ouimet CC, Björklund A (1989) Host afferents into intrastriatal transplants of fetal ventral mesencephalon. Exp Neurol 106:1-19.

Flecknell PA (1996) Laboratory animal anesthesia, Ed 2. London: Academic

Gates MA, Laywell ED, Fillmore H, Steindler DA (1996) Astrocytes and extracellular matrix following intracerebral transplantation of embryonic ventral mesencephalon or lateral ganglionic eminence. Neuroscience 74:579-597.

GrandPre T, Nakamura F, Vartanian T, Strittmatter SM (2000) Identification of the Nogo inhibitor of axon regeneration as a Reticulon protein. Nature 403:439-444

Haas CA, Rauch U, Thon N, Merten T, Deller T (1999) Entorhinal cortex lesion in adult rats induces the expression of the neural chondroitin sulfate proteoglycan neurocan in reactive astrocytes. J Neurosci 19:9953-9963.

Huang DW, McKerracher L, Braun PE, David S (1999) A therapeutic vaccine approach to stimulate axon regeneration in the adult mammalian spinal cord. Neuron 24:639-647.

Isacson O, Deacon TW (1996) Specific axon guidance factors persist in the adult brain as demonstrated by pig neuroblasts transplanted to the rat. Neuroscience 75:827-837.

Kim WG, Mohney RP, Wilson B, Jeohn GH, Liu B, Hong JS (2000) Regional difference in susceptibility to lipopolysaccharide-induced neurotoxicity in the rat brain: role of microglia. J Neurosci 20:6309-6316.

Krobert K, Lopez-Colberg I, Cunningham LA (1997) Astrocytes promote or impair the survival and function of embryonic ventral mesencephalon co-grafts: effects of astrocyte age and expression of recombinant brain-derived neurotrophic factor. Exp Neurol 145:511-523.

Labandeira-Garcia JL, Wictorin K, Cunningham ET, Björklund A (1991) Development of intrastriatal striatal grafts and their afferent innervation from the host. Neuroscience 42:407-426.

Laywell ED, Steindler DA (1991) Boundaries and wounds, glia and glycoconjugates. Ann NY Acad Sci 633:122-141.

Liu JP, Lauder JM (1992) Serotonin promotes region-specific glial influences on cultured serotonin and dopamine neurons. Glia 5:306-317.

Lu SY, Shipley MT, Norman AB, Sanberg PR (1991) Striatal, ventral mesencephalic and cortical transplants into the intact rat striatum: a neuroanatomical study. Exp Neurol 113:109-130.

Mamounas LA, Blue ME, Siuciak JA, Altar CA (1995) Brain-derived neurotrophic factor promotes the survival and sprouting of serotonergic axons in rat brain. J Neurosci 15:7929-7939.

Mamounas LA, Altar AC, Blue ME, Kaplan DR, Tessarollo L, Lyons EW (2000) BDNF promotes the regenerative sprouting, but not survival, of injured serotoninergic axons in the adult rat brain. J Neurosci 20:771-782.

McCarthy KD, De Vellis J (1980) Preparation of separate astroglial and 
oligodendroglial cell cultures from rat cerebral tissue. J Cell Biol 85:890-902.

McKeon RJ, Schreiber RC, Rudge JS, Silver J (1991) Reduction of neurite outgrowth in a model of glial scarring following CNS injury is correlated with the expression of inhibitory molecules on reactive astrocytes. J Neurosci 11:3398-3411.

McKerracher LJ, David S, Jackson DL, Kottis V, Dunn RJ, Braun PE (1994) Identification of myelin-associated glycoprotein as a major myelin-derived inhibitor of neurite growth. Neuron 13:805-811.

Morin F, Crevier C, Bouvier G, Lacaille J-C, Beaulieu C (1997) A fixation procedure for ultrastructural investigation of synaptic connections in resected human cortex. Brain Res Bull 44:205-210.

Moukhles H, Bosler O, Bolam JP, Vallée A, Umbriaco D, Geffard M, Doucet G (1997) Quantitative and morphometric data indicate precise cellular interactions between serotonin terminals and postsynaptic targets in rat substantia nigra. Neuroscience 76:1159-1171.

Mounir A, Chkirate M, Vallée A, Pierret P, Geffard M, Doucet G (1994) Host serotonin axons innervate intrastriatal ventral mesencephalic grafts after implantation in newborn rat. Eur J Neurosci 6:1307-1315.

Mukhopadhyay G, Doherty P, Walsh FS, Crocker PR, Filbin MT (1994) A novel role for myelin-associated glycoprotein as an inhibitor of axonal regeneration. Neuron 13:757-767.

Nothias F, Onténiente B, Geffard M, Peschanski M (1990) Dissimilar responses of adult thalamic monoaminergic and somatosensory afferent fibers to implantation of thalamic fetal cells. Neuroscience 37:353-366.

Oblinger MM, Das GD (1982) Connectivity of neural transplants in adult rats: analysis of afferents and efferents of neocortical transplants in the cerebellar hemisphere. Brain Res 249:31-49.

Pierret P, Quenneville N, Vandaele S, Abbaszadeh R, Lanctôt C, Crine P, Doucet G (1998a) Trophic and tropic effects of striatal astrocytes on cografted mesencephalic dopamine neurons and their axons. J Neurosci Res 51:23-40.

Pierret P, Vallée A, Bosler O, Dorais M, Moukhles H, Abbaszadeh R, Lepage Y, Doucet G (1998b) Serotonin axons of the neostriatum show a higher affinity for striatal than for ventral mesencephalic transplants: a quantitative study in adult and immature recipient rats. Exp Neurol 152:101-115.

Powell EM, Meiners S, DiProspero NA, Geller HM (1997) Mechanisms of astrocyte-directed neurite guidance. Cell Tissue Res 290:385-393.

Smith GM, Miller RH, Silver J (1986) Changing role of forebrain astro- cytes during development, regenerative failure, and induced regeneration upon transplantation. J Comp Neurol 251:23-43.

Soghomonian J-J, Doucet G, Descarries L (1987) Serotonin innervation in adult rat neostriatum. I. Quantified regional distribution. Brain Res 425:85-100.

Sørensen JC, Wanner-Olsen H, Tønder N, Danielsen E, Castro AJ, Zimmer J' (1990) Axotomized, adult basal forebrain neurons can innervate fetal frontal cortex grafts: a double fluorescent tracer study in the rat. Exp Brain Res 81:545-551.

Stein E, Tessier-Lavigne M (2001) Hierarchical organization of guidance receptors: silencing of netrin attraction by slit through a Robo/ DCC receptor complex. Science 291:1928-1938.

Steindler DA, O'Brien TF, Laywell E, Harrington K, Faissner A, Schachner M (1990) Boundaries during normal and abnormal brain development: in vivo and in vitro studies of glia and glycoconjugates. Exp Neurol 109:35-56.

Streit WJ, Kreutzberg GW (1987) Lectin binding by resting and reactive microglia. J Neurocytol 16:249-260.

Tessier-Lavigne M, Goodman CS (1996) The molecular biology of axon guidance. Science 274:1123-1133.

Van der Kooy D, Hattori T (1980) Dorsal raphe cells with collateral projections to the caudate-putamen and substantia nigra: a fluorescent retrograde double study in the rat. Brain Res 186:1-7.

Vickland H, Silver J (1996) The role of astrocytes in axons guidance during development and repair. In: Glial cell development: basic principles and clinical relevance (Jessen KR, Richardson WD, eds), pp 197-208. Oxford: BIOS Scientific.

Whitaker-Azmitia PM, Murphy R, Azmitia EC (1990) Stimulation of astroglial 5-HT1A receptors releases the serotoninergic growth factor, protein S-100, and alters astroglial morphology. Brain Res 528:155-158.

Wictorin K, Isacson O, Fischer W, Nothias F, Peschanski M, Björklund A (1988) Connectivity of striatal grafts implanted into the ibotenic acidlesioned striatum: 1. Subcortical afferents. Neuroscience 27:547-562.

Wictorin K, Lagenaur CF, Lund RD, Bjorklund A (1991) Efferent projections to the host brain from intrastriatal striatal mouse-to-rat grafts: time course and tissue-type specificity as revealed by a mouse-specific neuronal marker. Eur J Neurosci 3:86-101.

Xu XM, Guenard V, Kleitman N, Aebischer P, Bunge MB (1995) A combination of BDNF and NT-3 promotes supraspinal axonal regeneration into Schwann cell grafts in adult rat thoracic spinal cord. Exp Neurol 134:261-272. 